\title{
Accurate reproduction of strongly repulsive interatomic potentials
}

\section{Lehtola, Susi}

2020-03-05

Lehtola, S 2020 , ' Accurate reproduction of strongly repulsive interatomic potentials ',

Physical Review A, vol. 101 , no. 3 , 032504 . https://doi.org/10.1103/PhysRevA.101.032504

http://hdl.handle.net/10138/313899

https://doi.org/10.1103/PhysRevA.101.032504

unspecified

publishedVersion

Downloaded from Helda, University of Helsinki institutional repository.

This is an electronic reprint of the original article.

This reprint may differ from the original in pagination and typographic detail.

Please cite the original version. 


\title{
Accurate reproduction of strongly repulsive interatomic potentials
}

\author{
Susi Lehtola $\odot^{*}$ \\ Department of Chemistry, University of Helsinki, P.O. Box 55 (A. I. Virtasen aukio 1), FI-00014 University of Helsinki, Finland
}

(Received 29 December 2019; accepted 7 February 2020; published 5 March 2020)

\begin{abstract}
Knowledge of the repulsive behavior of potential energy curves $V(R)$ at $R \rightarrow 0$ is necessary for understanding and modeling irradiation processes of practical interest. $V(R)$ is in principle straightforward to obtain from electronic structure calculations; however, commonly used numerical approaches for electronic structure calculations break down in the strongly repulsive region due to the closeness of the nuclei. In this work, we show by comparison to fully numerical reference values that a recently developed procedure [S. Lehtola, J. Chem. Phys. 151, 241102 (2019)] can be employed to enable accurate linear combination of atomic orbitals calculations of $V(R)$ even at small $R$ by a study of the seven nuclear reactions $\mathrm{He}_{2} \rightleftharpoons \mathrm{Be}, \mathrm{HeNe} \rightleftharpoons \mathrm{Mg}$, $\mathrm{Ne}_{2} \rightleftharpoons \mathrm{Ca}, \mathrm{HeAr} \rightleftharpoons \mathrm{Ca}, \mathrm{MgAr} \rightleftharpoons \mathrm{Zn}, \mathrm{Ar}_{2} \rightleftharpoons \mathrm{Kr}$, and $\mathrm{NeCa} \rightleftharpoons \mathrm{Zn}$
\end{abstract}

DOI: 10.1103/PhysRevA.101.032504

\section{INTRODUCTION}

The interaction of high-energy particles with matter is typically modeled using pairwise potentials (see, e.g., Chap. 6 of [1]), as the dominant interactions are determined by the highly repulsive nuclear Coulomb barriers that are pairwise terms; see, e.g., [2] for a recent numerical demonstration for low-energy projectiles incident on copper surfaces. Most practical simulations employ the universal potential by Ziegler, Biersack, and Littmark [3] (ZBL) which is based on ThomasFermi calculations of the repulsive barrier. However, ThomasFermi theory has significant shortcomings; for instance, it is well known not to bind any molecules, and a method lacking these shortcomings like Hartree-Fock (HF) or densityfunctional theory $[4,5]$ (DFT) would certainly be more attractive.

$A b$ initio calculations of the diatomic potential energy curve (PEC), denoted here as $V_{A B}(R)$, are, however, challenging at small internuclear distances $R$ due to the closeness of the two nuclei. In contrast to chemistry at ambient conditions, even the innermost core electrons may be significantly affected by the interaction between the two atoms: for instance, in the $\mathrm{Ar}_{2} \rightleftharpoons \mathrm{Kr}$ nuclear reaction obtained as $R \rightarrow 0$, the two $[\mathrm{Ne}] 3 s^{2} 3 p^{6}$ electronic configurations of the argon atoms deform into the single [Ne] $3 s^{2} 3 p^{6} 4 s^{2} 3 d^{10} 4 p^{6}$ configuration of the krypton atom. An extremely flexible numerical approach must be used in order to describe such changes accurately, obviously disallowing the use of pseudopotential and frozencore approaches. Although some efforts for the $a b$ initio description of the Coulomb barrier have been made in the literature (see, e.g., [2,6-17], and references therein), the problem of facile computation of $V_{A B}(R)$ for $R \rightarrow 0$ remains still unsolved in the general case.

All-electron calculations are typically undertaken within the linear combination of atomic orbitals (LCAO) approach. However, also the LCAO approach fails in this case because

\footnotetext{
*susi.lehtola@alumni.helsinki.fi
}

the basis functions on the atoms $A$ and $B$ quickly become linearly dependent when $R \rightarrow 0$. Moreover, large atomic basis sets should be used in order to allow the necessary flexibility for the core orbitals to deform in presence of the other nucleus and its electrons. But, the more functions are included in the calculations, the more linear dependencies are generated when the nuclei start coinciding, and the calculations become numerically unstable as the basis set becomes ill behaved.

As always, fully numerical electronic structure calculations are one option (see [18] for a recent review). Here, the numerical basis set can always be chosen in such a way that linear dependencies do not arise even at small $R$. However, fully numerical approaches carry a much higher computational cost than that of LCAO calculations using, e.g., Gaussian basis sets, and may also be harder to set up; see the discussion in $[18,19]$. Moreover, fully numerical electronic structure programs are less developed than Gaussian-basis ones because the huge number of basis functions in a fully numerical approach may, e.g., make sophisticated convergence algorithms intractable [18], making it more difficult to carry out the wanted electronic structure calculations.

Despite the numerical problems encountered in standard LCAO approaches, it should be perfectly well possible to describe diatomic molecules using atomic basis sets even at small internuclear distances because at small $R$ the molecule looks like the compound atom that is especially easy to describe with atomic basis sets. This means that the problems in LCAO calculations should be circumventable by adopting a basis set that is adapted to the molecular geometry. (In contrast, significant distortions to the electronic structure of atoms and molecules can be observed, e.g., in strong magnetic fields as discussed in [20] and references therein, in which case LCAO calculations become unreliable.)

Because the electronic structure at $R \rightarrow 0$ may be quite far from those for which typical basis sets have been optimized, one can customize the basis set for the system by hand as in [7]. (Alternatively, one could also optimize a new basis set from scratch for the system.) However, given that this would lead to a different basis set for every molecule and for 
every molecular geometry, a systematic study of the repulsive potentials of all the elements in the periodic table would be faced with a gargantuan task for basis-set generation. For instance, the PECs for all 4186 diatomic molecules from $Z=1$ to 92 were calculated in [17] at internuclear distances ranging from $R=0.002 \AA$ to $R=1000 \AA$; this is only feasible with a fully automatic approach. (Convergence to the basis-set limit was not checked in [17], and we will show later in this work that the values are not converged.)

Issues with linear dependencies are encountered also in other applications of quantum chemistry. For instance, an accurate description of weakly bound anions may require the use of several shells of diffuse functions on each atom, making the molecular basis set ill behaved due to linear dependencies [21]. An approach for curing overcompleteness issues in the study of weakly bound anions with LCAO basis sets by pivoted Cholesky decompositions was recently proposed in [22]. Cholesky decomposition algorithms have a long history in quantum chemistry, starting with the decomposition of the two-electron integral tensor proposed over 40 years ago by Beebe and Linderberg [23] that has recently become popular due to efficient implementations afforded by modern computer architectures (see, e.g., [24,25]). The tractability of the Cholesky decomposition of the two-electron integrals relies on the set of basis function products $\chi_{i}(\boldsymbol{r}) \chi_{j}(\boldsymbol{r})$ being highly linearly dependent: due to these dependencies, the number of Cholesky vectors of the two-electron integrals tensor grows only linearly with system size. Also method-specific variants related to the two-electron integrals tensor decomposition have been suggested (see [26] for a review). The construction of localized orbitals by the Cholesky decomposition of the LCAO one-electron density matrix has also been proposed [27].

The mathematical closeness of the Cholesky decomposition of the two-electron integrals tensor, $(i j \mid k l)=$ $\sum_{P} L_{(i j)}^{P} L_{(k l)}^{P}$ in chemists' notation, to resolution-of-theidentity methods [28], $(i j \mid k l) \approx \sum_{A B}(i j \mid A)(A \mid B)^{-1}(B \mid k l)$, led to a black-box procedure for the formation of auxiliary basis sets $[29,30]$, in which the pivot index of the Cholesky decomposition of the two-electron integrals is used to determine the auxiliary functions; the resulting set of auxiliary functions is better behaved than the set of basis function products. Building on the work of [29,30], we proposed curing significant linear dependencies in overcomplete basis sets by the extraction of a well-behaved subset of the basis functions using a pivoted Cholesky procedure on the overlap matrix $S_{\mu \nu}=\langle\mu \mid \nu\rangle$ to a predefined threshold $\tau$ [22]; this yields an optimal approximation for the original overcomplete $S$ [31]. Electronic structure calculations can be carried out in the basis set defined by the set of pivot functions without problems. Before [22], Cholesky decompositions of the overlap matrix appear to have only been used in the full, unpivoted form [32-34] that is not safe for ill-conditioned matrices $S$.

In this work, we show that the partial Cholesky decomposition algorithm proposed in [22] for calculations with basis sets containing a large number of linearly dependent diffuse functions presents a solution to the present problem of the calculation of strongly repulsive interatomic potentials by allowing the use of standard atomic basis sets even at $R \rightarrow 0$ since the basis function degeneracies that would otherwise prevent reliable electronic structure calculations from taking place are cleaned away automatically.

As our aim is simply to prove that the basis set limit can be reached without problem even at tiny values of $R$, we have chosen to study a set of seven nuclear reactions involving only closed-shell atoms: $\mathrm{He}_{2} \rightleftharpoons \mathrm{Be}, \mathrm{HeNe} \rightleftharpoons \mathrm{Mg}, \mathrm{Ne}_{2} \rightleftharpoons$ $\mathrm{Ca}, \mathrm{HeAr} \rightleftharpoons \mathrm{Ca}, \mathrm{MgAr} \rightleftharpoons \mathrm{Zn}, \mathrm{Ar}_{2} \rightleftharpoons \mathrm{Kr}$, and $\mathrm{NeCa} \rightleftharpoons \mathrm{Zn}$. We show that the suggested Cholesky procedure reproduces fully numerical HF reference values for the reactions, while the values reported in [17] are not converged for small $R$. The present case is much more challenging than that of [22] as, e.g., in $\mathrm{He}_{2}, \mathrm{Ne}_{2}$, and $\mathrm{Ar}_{2}$ all basis functions of an atom become fully degenerate with the basis function of the other atom for $R \rightarrow 0$. Our calculations will be described in Sec. II and our results reported in Sec. III. The work is briefly summarized and discussed in Sec. IV. Atomic units are used throughout the paper.

\section{COMPUTATIONAL DETAILS}

The PEC for atoms $A$ and $B$ is defined as

$$
V_{A B}(R)=E_{\mathrm{tot}}^{A+B}(R)-E_{\mathrm{el}}^{A}-E_{\mathrm{el}}^{B},
$$

where $E_{\mathrm{tot}}^{A+B}(R)$ is the total energy from the electronic structure calculation for the nuclei $A$ and $B$ separated by a distance of $R$, and $E_{\mathrm{el}}^{A}$ and $E_{\mathrm{el}}^{B}$ are the electronic energies of the noninteracting atoms, respectively. The total energy $E_{\mathrm{tot}}^{A+B}$ can be decomposed into a sum of the electronic energy $E_{\mathrm{el}}^{A+B}(R)$ and the nuclear repulsion energy $E_{\text {nuc }}^{A+B}(R)$. Since the electronic energy of the compound atom $(A+B)$ is finite, $E_{\mathrm{tot}}^{A+B}(R)$ behaves asymptotically as $E_{\mathrm{tot}}^{A+B}(R) \approx E_{\mathrm{nuc}}^{A+B}(R)=Z_{A} Z_{B} R^{-1}$ for small $R$. Because $V_{A B}(R)$ thus diverges for small $R$, it is typical to report the PEC in terms of a screening function

$$
\Phi_{A B}(R)=\frac{V_{A B}(R)}{E_{\mathrm{nuc}}^{A+B}(R)}=\frac{R V_{A B}(R)}{Z_{A} Z_{B}}
$$

as it is more easily manipulable, having the limits $\Phi_{A B}(0)=1$ and $\Phi_{A B}(\infty)=0$.

Although the procedure of [22] can be used with any type of atomic basis set (see [18] for a review thereof), Gaussian basis sets are employed in this work. Furthermore, while the approach of [22] can also be applied to density functional or post-HF calculations, the HF level of theory is used in this work as it has been found to be sufficient for the reproduction of repulsive potentials [10].

The ERKALE program [35,36] is used for the Gaussianbasis calculations. The nuclei $A$ and $B$ are placed in the ERKALE calculations along the $z$ axis at $(0,0,-R / 2)$ and $(0,0, R / 2)$, respectively, along with their atomic basis functions. Next, in order to be able to describe the compound atom $(A+B)$ limit, basis functions for the compound atom are included in the calculation; placing the compound nucleus at the center of charge at $\left(0,0,\left(Z_{B}-Z_{A}\right) R /\left[2\left(Z_{B}+Z_{A}\right)\right]\right)$ leads to a vanishing dipole moment of the nuclear charge distribution, and hopefully a more accurate calculation. Once the basis functions for the compound nucleus have been added, the construction of the one-electron basis $\{|\mu\rangle\}$ is complete; however, by this stage the basis set is likely overcomplete. 
TABLE I. Values of screening function $\Phi(R)$ computed at various points $R$ (value in $\AA$ given on the first row) with the fully numerical HELFEM program. The Gaussian-basis-set truncation errors $\Delta$ basis $=\Phi^{\text {basis }}(R)-\Phi^{\text {reference }}(R)$ of the un-pc- $n$ and UGBS basis sets are also shown; these calculations were done with ERKALE.

\begin{tabular}{|c|c|c|c|c|c|c|c|c|c|c|c|}
\hline & & $10^{-3}$ & $10^{-2.5}$ & $10^{-2}$ & $10^{-1.5}$ & $10^{-1.25}$ & $10^{-1}$ & $10^{-0.75}$ & $10^{-0.5}$ & $10^{-0.25}$ & $10^{0}$ \\
\hline \multirow[t]{5}{*}{$\mathrm{He}_{2}$} & HELFEM & 0.99582 & 0.98678 & 0.95831 & 0.87089 & 0.77943 & 0.64265 & 0.47484 & 0.34264 & 0.21805 & 0.07205 \\
\hline & $\Delta$ un-pc-1 & 0.00000 & -0.00001 & -0.00005 & -0.00007 & 0.00027 & 0.00095 & 0.00068 & 0.00023 & 0.00162 & 0.00011 \\
\hline & $\Delta$ un-pc-2 & 0.00000 & 0.00000 & 0.00000 & 0.00004 & 0.00006 & 0.00008 & 0.00007 & 0.00005 & 0.00041 & -0.00005 \\
\hline & $\Delta$ un-pc-3 & 0.00000 & 0.00000 & 0.00000 & 0.00000 & 0.00000 & 0.00000 & 0.00001 & 0.00002 & 0.00002 & 0.00000 \\
\hline & $\Delta \mathrm{UGBS}$ & 0.00000 & 0.00000 & 0.00000 & 0.00000 & 0.00000 & 0.00000 & 0.00001 & 0.00006 & 0.00021 & 0.00022 \\
\hline \multirow[t]{5}{*}{$\mathrm{HeNe}$} & HELFEM & 0.99356 & 0.97966 & 0.93658 & 0.81686 & 0.71223 & 0.57716 & 0.40213 & 0.21037 & 0.09810 & 0.03762 \\
\hline & $\Delta$ un-pc-1 & -0.00001 & -0.00003 & -0.00007 & 0.00000 & 0.00013 & -0.00007 & -0.00004 & 0.00016 & -0.00022 & -0.00111 \\
\hline & $\Delta$ un-pc-2 & 0.00000 & 0.00000 & 0.00000 & 0.00006 & 0.00006 & 0.00009 & 0.00024 & 0.00037 & 0.00014 & -0.00010 \\
\hline & $\Delta$ un-pc-3 & 0.00000 & 0.00000 & 0.00000 & 0.00000 & 0.00001 & 0.00004 & 0.00006 & 0.00003 & 0.00002 & 0.00000 \\
\hline & $\triangle \mathrm{UGBS}$ & 0.00000 & 0.00000 & 0.00000 & 0.00000 & 0.00001 & 0.00008 & 0.00031 & 0.00085 & 0.00120 & 0.00031 \\
\hline \multirow[t]{5}{*}{$\mathrm{Ne}_{2}$} & & & & 0.92326 & & & 04 & & 0.20790 & & 0.02656 \\
\hline & $\Delta$ un-pc-1 & 0.00000 & -0.00001 & 0.00001 & -0.00001 & 0.00008 & 0.00053 & 0.00065 & 0.00029 & -0.00007 & -0.00035 \\
\hline & $\Delta$ un-pc-2 & 0.00000 & 0.00000 & 0.00000 & 0.00001 & 0.00006 & 0.00009 & 0.00007 & 0.00009 & 0.00007 & -0.00001 \\
\hline & $\Delta$ un-pc-3 & 0.00000 & 0.000 & 0.00000 & 0.00000 & 0.000 & 0.00001 & & & & 0.00001 \\
\hline & $\Delta \mathrm{UGBS}$ & 0.00000 & 0.00000 & 0.00000 & 0.00000 & 0.00000 & 0.0 & 2 & 0.00 & 0. & 0.00011 \\
\hline \multirow[t]{5}{*}{$\mathrm{HeAr}$} & HELFEM & 0.99228 & 0.97569 & 0.92539 & 0.79716 & 0.69117 & 0.54811 & 0.38703 & 0.24271 & 0.10196 & 0.04028 \\
\hline & $\Delta$ un-pc-1 & 0.00000 & 0.00000 & 0.00001 & 0.00012 & 0.00029 & 0.00058 & 0.00060 & 0.00043 & 0.00 & 0.00001 \\
\hline & $\Delta$ un-pc-2 & 0.00000 & 0.00000 & 0.00001 & 0.00008 & 0.00008 & 0.00019 & 0.00028 & 0.00020 & 0.00 & 0.00001 \\
\hline & $\Delta$ un-pc-3 & 0.00000 & 0.00000 & 0.00000 & 0.00001 & 0.00004 & 0.00010 & 0.00011 & 0.00006 & 0.00002 & 0.00001 \\
\hline & $\Delta \mathrm{UGBS}$ & 0.00000 & 0.00000 & 0.00000 & 0.00001 & 0.00004 & 0.00016 & 0.00038 & 0.00043 & 0.00084 & 0.00083 \\
\hline \multirow[t]{5}{*}{$\mathrm{MgAr}$} & HELFEM & 0.99081 & 0.97114 & 0.91299 & 0.77196 & 0.65409 & 0.50202 & 0.33521 & 0.17926 & 0.07422 & 0.02229 \\
\hline & $\Delta$ un-pc-1 & 0.00000 & 0.00000 & 0.00000 & 0.00003 & 0.00006 & 0.00008 & 0.00024 & 0.00091 & 0.00032 & 0.00018 \\
\hline & $\Delta$ un-pc-2 & 0.00000 & 0.00000 & 0.00000 & 0.00001 & 0.00002 & 0.00004 & 0.00017 & 0.00056 & 0.00012 & 0.00009 \\
\hline & $\Delta$ un-pc-3 & 0.00000 & 0.00000 & 0.00000 & 0.00000 & 0.00000 & 0.00002 & 0.00009 & 0.00018 & 0.00005 & 0.00002 \\
\hline & $\Delta \mathrm{UGBS}$ & 0.00000 & 0.00000 & 0.00000 & 0.00000 & 0.00001 & 0.00003 & 0.00020 & 0.00086 & 0.00029 & 0.00025 \\
\hline \multirow[t]{6}{*}{$\mathrm{Ar}_{2}$} & HELFEM & 0.99011 & 0.96900 & 0.90749 & 0.76090 & 0.63761 & 0.48451 & 0.31334 & 0.17568 & 0.07255 & 0.02137 \\
\hline & $\Delta \mathrm{LDA}^{\mathrm{a}}$ & 0.00001 & 0.00003 & 0.00009 & 0.00012 & 0.00017 & 0.00028 & 0.00073 & -0.00130 & 0.00174 & no data \\
\hline & $\Delta$ un-pc-1 & 0.00000 & 0.00000 & 0.00000 & 0.00003 & 0.00005 & 0.00013 & 0.00089 & 0.00158 & 0.00290 & 0.00155 \\
\hline & $\Delta$ un-pc-2 & 0.00000 & 0.00000 & 0.00000 & 0.00001 & 0.00002 & 0.00009 & 0.00080 & 0.00117 & 0.00115 & 0.00066 \\
\hline & $\Delta$ un-pc-3 & 0.00000 & 0.00000 & 0.00000 & 0.00000 & 0.00001 & 0.00006 & 0.00055 & 0.00029 & 0.00010 & 0.00030 \\
\hline & $\Delta \mathrm{UGBS}$ & 0.00000 & 0.00000 & 0.00000 & 0.00000 & 0.00001 & 0.00009 & 0.00078 & 0.00141 & 0.00275 & 0.00078 \\
\hline \multirow[t]{5}{*}{$\mathrm{NeCa}$} & HELFEM & 0.99082 & 0.97117 & 0.91310 & 0.77230 & 0.65438 & 0.50203 & 0.33509 & 0.17662 & 0.07093 & 0.01768 \\
\hline & $\Delta$ un-pc-1 & 0.00000 & 0.00000 & 0.00002 & 0.00005 & 0.00012 & 0.00032 & 0.00057 & 0.00061 & 0.00023 & 0.00008 \\
\hline & $\Delta$ un-pc-2 & 0.00000 & 0.00000 & 0.00000 & 0.00002 & 0.00005 & 0.00007 & 0.00016 & 0.00026 & 0.00011 & 0.00004 \\
\hline & $\Delta$ un-pc-3 & 0.00000 & 0.00000 & 0.00000 & 0.00000 & 0.00001 & 0.00002 & 0.00006 & 0.00007 & 0.00002 & 0.00001 \\
\hline & $\triangle \mathrm{UGBS}$ & 0.00000 & 0.00000 & 0.00000 & 0.00000 & 0.00001 & 0.00003 & 0.00021 & 0.00095 & 0.00047 & 0.00049 \\
\hline
\end{tabular}

aThe data for $\mathrm{Ar}_{2}$ also include the differences between the LDA and HF screening functions' reference values $\Delta \mathrm{LDA}=\Phi^{\mathrm{LDA}}(R)-\Phi^{\mathrm{HF}}(R)$, both of which have been computed with HELFEM.

Next, the overlap matrix $S_{\mu \nu}=\langle\mu \mid \nu\rangle$, its eigenvalues $\lambda_{i}$, and its reciprocal condition number

$$
r=\frac{\lambda_{\min }}{\lambda_{\max }}
$$

are computed. If the basis set is found to be overcomplete, i.e., $r$ is found to be smaller than the machine epsilon, the Cholesky procedure of [22] is used to regularize the molecular basis set. The procedure uses a pivoted Cholesky decomposition to pick a subset of the basis functions $\{|\mu\rangle\}$ that span all of the functions in the original basis set up to a predefined threshold. The resulting reduced-size basis is numerically well conditioned, and poses no problems to electronic structure calculations which then proceed as usual. The procedure is implemented as a modification [22] to the canonical orthogonalization method [37]; a Cholesky threshold of $10^{-7}$ and a linear dependence threshold of $10^{-5}$ are used in this work. As the basis set is normalized, $S_{\mu \mu}=1$, the first function of the Cholesky procedure will be the first function in the basis set. However, since diffuse basis functions may be representable as superpositions of more localized basis functions while the converse is unlikely to be the case, the basis functions are reorganized from tight to diffuse before the Cholesky procedure. This procedure was found to yield more compact reduced basis sets in [22].

The screening function $\Phi(R)$ is computed with ERKALE on a logarithmic grid consisting of 121 points ranging from $R=10^{-5} \AA$ to $R=10 \AA$. The Gaussian-basis values are then compared to a set of fully numerical reference values obtained with the HELFEM program $[19,38,39]$. The superposition of atomic potentials (SAP) initial guess [40] is used in all ERKALE and HELFEM calculations in combination with local exchange potentials recently determined at the complete basis-set limit [41]. The SAP guess correctly includes the 
significant Pauli repulsion between the electrons on the two nuclei at small $R$ in contrast to its commonly used alternatives discussed in [40], thus leading to faster convergence of the self-consistent field procedure.

Only singlet $\Sigma$ wave functions are considered in this work, in analogy to [10]. In the cases of $\mathrm{He}_{2} \rightleftharpoons \mathrm{Be}, \mathrm{HeNe} \rightleftharpoons \mathrm{Mg}$, $\mathrm{Ne}_{2} \rightleftharpoons \mathrm{Ca}$, and $\mathrm{HeAr} \rightleftharpoons \mathrm{Ca}$, the large- $R$ and small- $R$ wave functions have the same electronic configurations: two occupied $\sigma$ orbitals for $\mathrm{He}_{2}$ and $\mathrm{Be}$, four $\sigma$ and one $\pi$ orbital for $\mathrm{HeNe}$ and $\mathrm{Mg}$, and six $\sigma$ and two $\pi$ orbitals for $\mathrm{Ne}_{2}$, $\mathrm{Ca}$, and $\mathrm{HeAr}$, each $\sigma$ and $\pi$ orbital fitting two and four electrons, respectively [18]. For the heavier systems, $\mathrm{MgAr} \rightleftharpoons$ $\mathrm{Zn}, \mathrm{Ar}_{2} \rightleftharpoons \mathrm{Kr}$, and $\mathrm{NeCa} \rightleftharpoons \mathrm{Zn}$, the electronic configurations are different at small $R$ and at large $R$, and both states were calculated: nine $\sigma$ and three $\pi$ in $\mathrm{MgAr}$ and $\mathrm{NeCa}$; seven $\sigma$, three $\pi$ and one $\delta$ orbital in $\mathrm{Zn}$; ten $\sigma$ and four $\pi$ orbitals in $\mathrm{Ar}_{2}$; and eight $\sigma$, four $\pi$ and one $\delta$ orbital in $\mathrm{Kr}, \delta$ orbitals likewise fitting four electrons [18]. The values reported correspond to the lower state in each case; for instance, the $\mathrm{Kr}$ configuration is lower in $\mathrm{Ar}_{2}$ for $R \lesssim 0.56 \AA$, the state crossing depending on the used basis set.

\section{RESULTS}

Very accurate LCAO calculations can be performed both at small $R$ and at large $R$, as in the former case a single expansion center is sufficient, and as in the latter the basis functions on the two centers do not develop strong linear dependencies. For this reason, we start off in Table I by comparing the values of the screening function $\Phi(R)$ at intermediate values of $R$ for the decontracted double- to quadruple- $\zeta$ pc- $n$ basis sets [42] (denoted as un-pc-1, un-pc-2, and un-pc-3, respectively) as well as for the universal Gaussian basis set [43] (UGBS) to fully numerical reference values.

Examination of the data in Table I shows that good results are already obtained with the double- $\zeta$ un-pc- 1 basis set, while the UGBS basis set appears to reproduce values that are

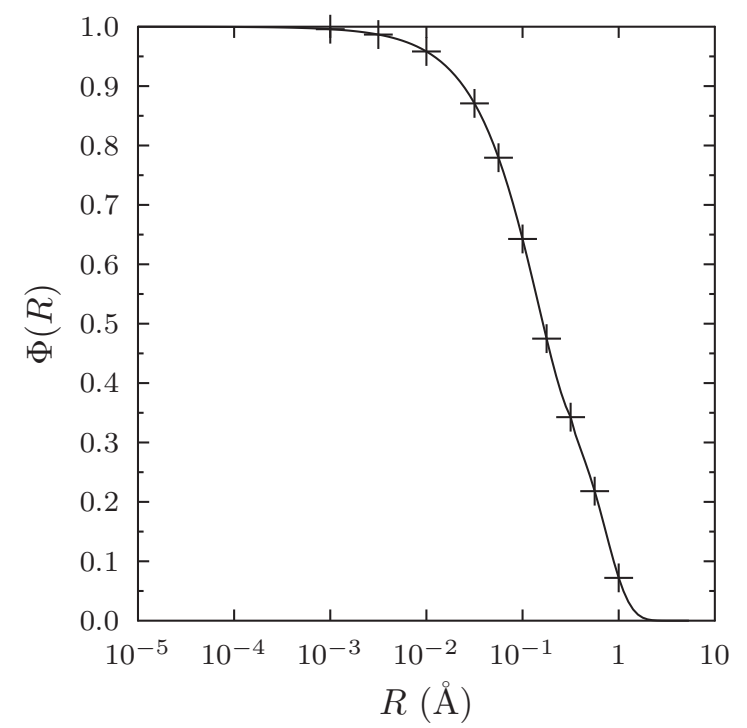

FIG. 1. UGBS screening function for the $\mathrm{He}_{2} \rightleftharpoons \mathrm{Be}$ reaction with fully numerical reference values $(+)$.

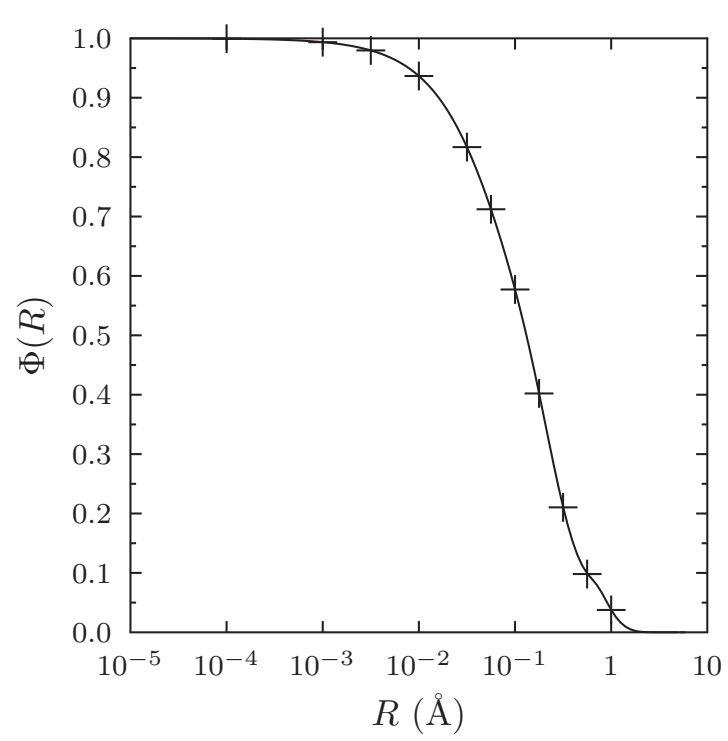

FIG. 2. UGBS screening function for the $\mathrm{HeNe} \rightleftharpoons \mathrm{Mg}$ reaction with fully numerical reference values $(+)$.

in-between those of the triple- $\zeta$ un-pc- 2 and the quadruple- $\zeta$ un-pc-3 basis set at small $R$. This suggests that the screening function is insensitive to polarization functions at small $R$; however, some polarization effects are already described by the compound nucleus basis functions included at the center of charge. As the UGBS basis set is available for most of the periodic table and equivalent atomic basis sets can be easily generated (see [44]), we choose the UGBS basis set for the rest of the work.

To confirm the finding of [10] that the screening function has a negligible dependence on the employed level of theory, we also report fully numerical reference values for $\mathrm{Ar}_{2}$ calculated with HELFEM using the local density approximation (LDA), in which the local exchange functional $[45,46]$ is combined with the Vosko-Wilk-Nusair correlation functional

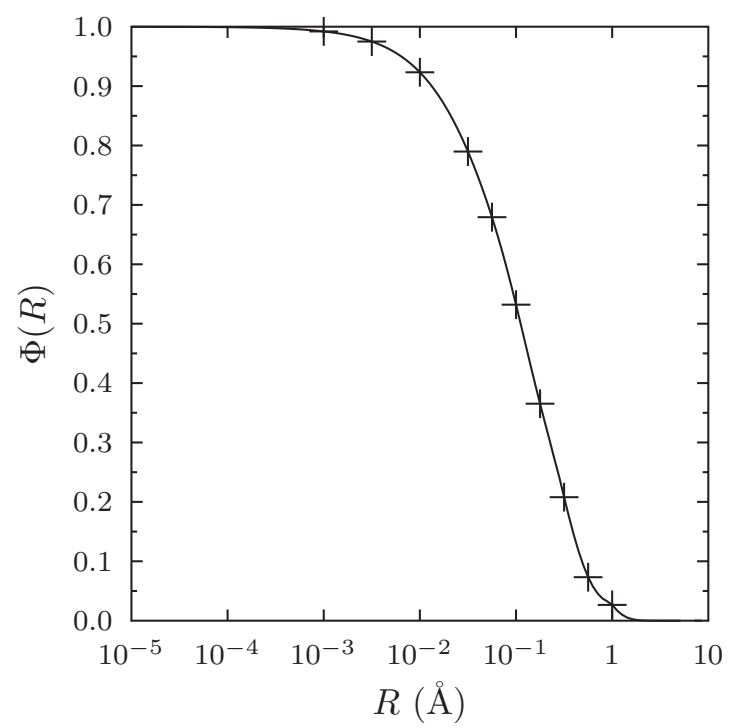

FIG. 3. UGBS screening function for the $\mathrm{Ne}_{2} \rightleftharpoons \mathrm{Ca}$ reaction with fully numerical reference values $(+)$. 


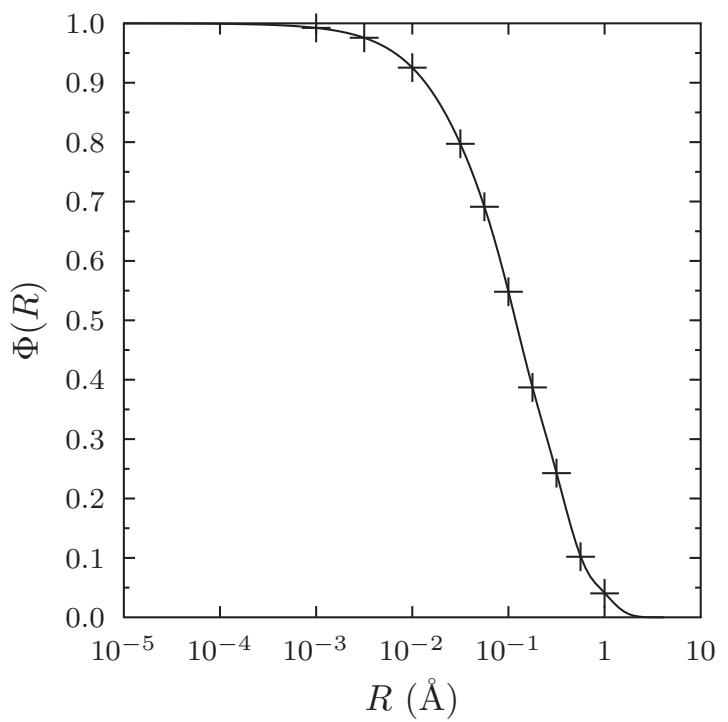

FIG. 4. UGBS screening function for the $\mathrm{HeAr} \rightleftharpoons \mathrm{Ca}$ reaction with fully numerical reference values $(+)$.

[47] as in [10]. The differences of the HF and LDA screening functions are only seen in the third decimal, confirming that HF or DFT is suitable for the present purposes.

The screening functions for the seven nuclear reactions computed with the UGBS basis set are shown in Fig. 1 for $\mathrm{He}_{2} \rightleftharpoons \mathrm{Be}$, Fig. 2 for $\mathrm{HeNe} \rightleftharpoons \mathrm{Mg}$, Fig. 3 for $\mathrm{Ne}_{2} \rightleftharpoons \mathrm{Ca}$, Fig. 4 for $\mathrm{HeAr} \rightleftharpoons \mathrm{Ca}$, Fig. 5 for $\mathrm{MgAr} \rightleftharpoons \mathrm{Zn}$, Fig. 6 for $\mathrm{Ar}_{2} \rightleftharpoons \mathrm{Kr}$, and Fig. 7 for $\mathrm{NeCa} \rightleftharpoons \mathrm{Zn}$. The curves are smooth and the agreement with fully numerical reference values is superb in all cases.

All of these reactions have also been studied in [17] (with the LDA approach of [10]) [48]. However, out of the seven reactions currently examined, Ref. [17] only reports data for $\mathrm{Ar}_{2} \rightleftharpoons \mathrm{Kr}$. A comparison to the UGBS results and fully numerical HF and LDA reference values is shown in Fig. 8.

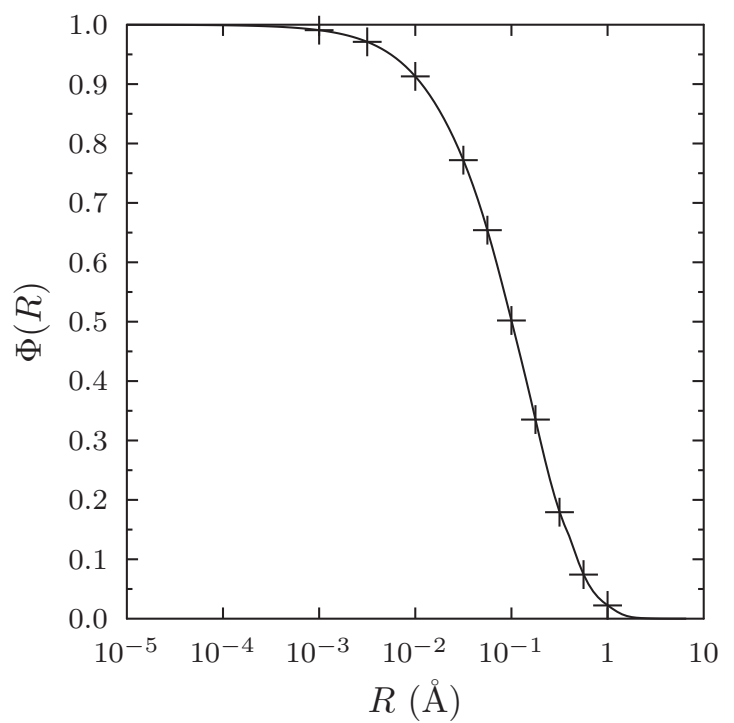

FIG. 5. UGBS screening function for the $\mathrm{MgAr} \rightleftharpoons \mathrm{Zn}$ reaction with fully numerical reference values $(+)$.

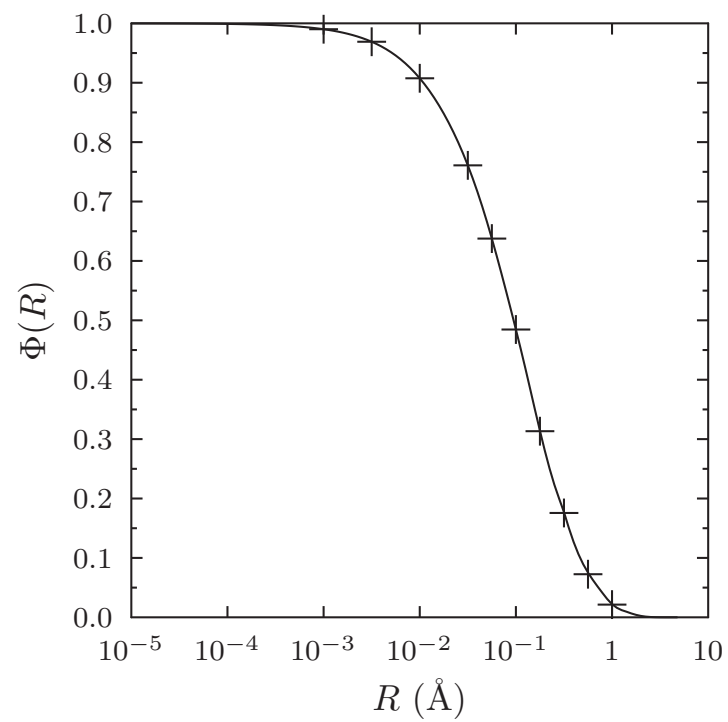

FIG. 6. UGBS screening function for the $\mathrm{ArAr} \rightleftharpoons \mathrm{Kr}$ reaction with fully numerical reference values $(+)$.

The data from [17] agree with the present values at large $R$, but discrepancies are visible for $R<0.1 \AA$. The UGBS data for HF agree with the fully numerical HF and LDA reference data, indicating that an insufficient basis set was used in [17].

\section{SUMMARY AND DISCUSSION}

We have shown by comparison to fully numerical HartreeFock reference values that accurate potential energy curves can be reproduced with linear combination of atomic orbitals (LCAO) calculations even in the strongly repulsive region at small internuclear distances, where even the core orbital basis functions become fully linearly dependent, by using a recently suggested procedure [22] to eliminate linear dependencies from the basis set. As LCAO calculations are faster and easier

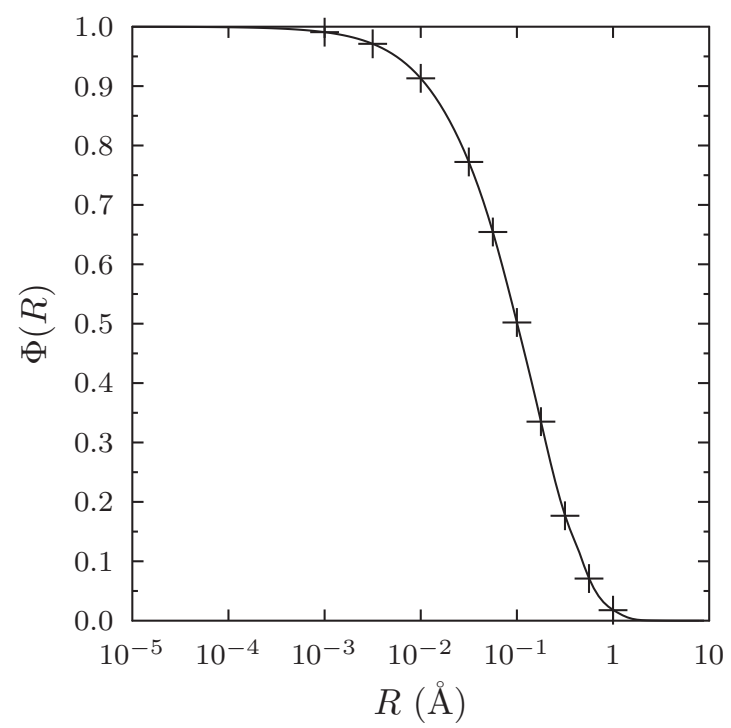

FIG. 7. UGBS screening function for the $\mathrm{NeCa} \rightleftharpoons \mathrm{Zn}$ reaction with fully numerical reference values $(+)$. 


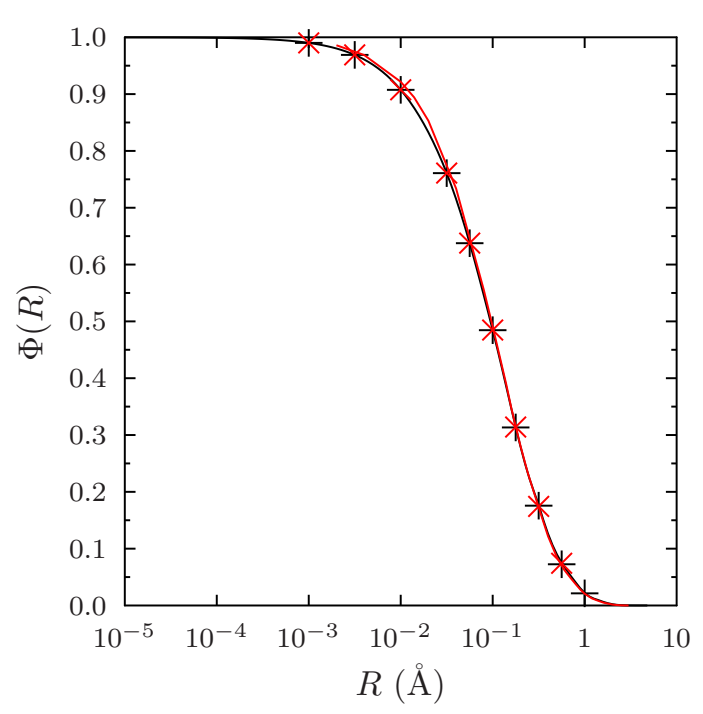

FIG. 8. Comparison of the $\mathrm{ArAr} \rightleftharpoons \mathrm{Kr}$ UGBS/Hartree-Fock screening function (black line) against LDA data from [17] (red line). Fully numerical Hartree-Fock (black + ) as well as LDA (red $\times$ ) reference values are also shown.

to run than fully numerical ones, the automated procedure of this work enables the systematical calculation of screening functions along the lines of [17] but with guaranteed accuracy.

Although this study has been limited to diatomic molecules, the underlying Cholesky decomposition has already been shown to work for polyatomic molecules [22], making the calculation of repulsive many-body potentials also possible.

The facile computation of the repulsive barrier afforded by the present method should make it easier to study various irradiation processes, in which the purely repulsive part of the potential plays a pivotal role. For instance, defect formation and migration in materials subjected to particle bombardment is determined purely by the repulsive part of the potential [15], and accounting for this kind of radiation damage is an important aspect in the design of radiation shielding materials of fusion reactors $[49,50]$.

This study has been limited to nonrelativistic calculations on light, closed-shell atoms. As relativistic effects increase rapidly in $Z$ [51,52], they are more important at the compound nucleus limit $R \rightarrow 0$ than at large $R$. Note also that in contrast to usual applications to chemistry, the screening function merits from no systematic error cancellation from the subtraction of atomic energies. The present procedure can, however, be straightforwardly extended to relativistic methods as well, making it possible to model the relativistic effects. Open-shell atoms as well as relativistic effects will be visited in future work.

Note added in proof. Instead of initializing the partial Cholesky decomposition by arranging the basis functions from tight to diffuse as done in the present work and [22], it appears that a simpler approach is also possible. Arranging the basis functions in $\mathbf{S}$ in order of increasing values of $s_{i}=\sum_{j \neq i}\left|S_{i j}\right|$ should provide the best starting point for the Cholesky procedure, as this will ensure that the functions with the smallest amount of overlap with other functions are used to seed the Cholesky decomposition. According to the Gershgorin circle theorem, $s_{i}$ provides a bound to the eigenvalue corresponding to the $i$ :th basis function; $s_{i} \approx 0$ means the basis function is almost an eigenvector of $\mathbf{S}$ with unit eigenvalue.

\section{ACKNOWLEDGMENTS}

I thank K. Nordlund and D. Sundholm for discussions on repulsive potentials. This work has been supported by the Academy of Finland (Suomen Akatemia) through Project No. 311149. Computational resources provided by CSC-It Center for Science Ltd (Espoo, Finland) and the Finnish Grid and Cloud Infrastructure (persistent identifier urn:nbn:fi:researchinfras-2016072533) are gratefully acknowledged.
[1] P. Sigmund, Particle Penetration and Radiation Effects Volume 2-Penetration of Atomic and Molecular Ions, Springer Series in Solid-State Sciences, Vol. 179 (Springer, Cham, 2014), p. 617

[2] M. A. Karolewski, Nucl. Instrum. Methods Phys. Res., Sect. B 256, 354 (2007).

[3] J. F. Ziegler, U. Littmark, and J. P. Biersack, The Stopping and Range of Ions in Solids (Pergamon, New York, 1985).

[4] P. Hohenberg and W. Kohn, Phys. Rev. 136, B864 (1964).

[5] W. Kohn and L. J. Sham, Phys. Rev. 140, A1133 (1965).

[6] N. H. Sabelli, M. Kantor, R. Benedek, and T. L. Gilbert, J. Chem. Phys. 68, 2767 (1978).

[7] N. H. Sabelli, R. Benedek, and T. L. Gilbert, Phys. Rev. A 20, 677 (1979).

[8] J. Keinonen, A. Kuronen, P. Tikkanen, H. G. Börner, J. Jolie, S. Ulbig, E. G. Kessler, R. M. Nieminen, M. J. Puska, and A. P. Seitsonen, Phys. Rev. Lett. 67, 3692 (1991).

[9] J. Keinonen, A. Kuronen, K. Nordlund, R. M. Nieminen, and A. P. Seitsonen, Nucl. Instrum. Methods Phys. Res., Sect. B 88, 382 (1994).
[10] K. Nordlund, N. Runeberg, and D. Sundholm, Nucl. Instrum. Methods Phys. Res., Sect. B 132, 45 (1997).

[11] J. M. Pruneda and E. Artacho, Phys. Rev. B 70, 035106 (2004).

[12] V. Kuzmin, Nucl. Instrum. Methods Phys. Res., Sect. B 249, 13 (2006).

[13] M. A. Karolewski, Nucl. Instrum. Methods Phys. Res., Sect. B 243, 43 (2006).

[14] V. Kuzmin, Surf. Coat. Technol. 201, 8388 (2007).

[15] N. Juslin and K. Nordlund, J. Nucl. Mater. 382, 143 (2008).

[16] M. A. Karolewski, Radiat. Eff. Defects Solids 167, 666 (2012).

[17] A. N. Zinoviev and K. Nordlund, Nucl. Instrum. Methods Phys. Res., Sect. B 406, 511 (2017).

[18] S. Lehtola, Int. J. Quantum Chem. 119, e25968 (2019).

[19] S. Lehtola, Int. J. Quantum Chem. 119, e25944 (2019).

[20] S. Lehtola, M. Dimitrova, and D. Sundholm, Mol. Phys. 118, e1597989 (2020).

[21] J. M. Herbert, Rev. Comput. Chem. 28, 391 (2015).

[22] S. Lehtola, J. Chem. Phys. 151, 241102 (2019). 
[23] N. H. F. Beebe and J. Linderberg, Int. J. Quant. Chem. 12, 683 (1977).

[24] S. D. Folkestad, E. F. Kjønstad, and H. Koch, J. Chem. Phys. 150, 194112 (2019).

[25] X. Feng, E. Epifanovsky, J. Gauss, and A. I. Krylov, J. Chem. Phys. 151, 014110 (2019).

[26] F. Aquilante, L. Boman, J. Boström, H. Koch, R. Lindh, A. S. de Merás, and T. B. Pedersen, in Linear-Scaling in Technical Computing in Chemical Physics, Vol. 13 (Springer, Dordrecht, 2011), pp. 301-343.

[27] F. Aquilante, T. B. Pedersen, A. Sánchez de Merás, and H. Koch, J. Chem. Phys. 125, 174101 (2006).

[28] O. Vahtras, J. Almlöf, and M. W. Feyereisen, Chem. Phys. Lett. 213, 514 (1993).

[29] F. Aquilante, R. Lindh, and T. B. Pedersen, J. Chem. Phys. 127, 114107 (2007).

[30] F. Aquilante, L. Gagliardi, T. B. Pedersen, and R. Lindh, J. Chem. Phys. 130, 154107 (2009).

[31] H. Harbrecht, M. Peters, and R. Schneider, Appl. Numer. Math. 62, 428 (2012).

[32] J. M. Millam and G. E. Scuseria, J. Chem. Phys. 106, 5569 (1997).

[33] M. Challacombe, J. Chem. Phys. 110, 2332 (1999).

[34] Y. Shao, C. Saravanan, M. Head-Gordon, and C. A. White, J. Chem. Phys. 118, 6144 (2003).
[35] J. Lehtola, M. Hakala, A. Sakko, and K. Hämäläinen, J. Comput. Chem. 33, 1572 (2012).

[36] S. Lehtola, ERKALE-HF/DFT from Hel.

[37] P.-O. Löwdin, Adv. Phys. 5, 1 (1956).

[38] S. Lehtola, Int. J. Quantum Chem. 119, e25945 (2019).

[39] S. Lehtola, HELFEM-Finite element methods for electronic structure calculations on small systems.

[40] S. Lehtola, J. Chem. Theory Comput. 15, 1593 (2019).

[41] S. Lehtola, Phys. Rev. A 101, 012516 (2020).

[42] F. Jensen, J. Chem. Phys. 115, 9113 (2001).

[43] E. V. R. de Castro and F. E. Jorge, J. Chem. Phys. 108, 5225 (1998).

[44] S. Lehtola, arXiv:2001.04224.

[45] F. Bloch, Z. Phys. 57, 545 (1929).

[46] P. A. M. Dirac, Math. Proc. Cambridge Philos. Soc. 26, 376 (1930).

[47] S. H. Vosko, L. Wilk, and M. Nusair, Can. J. Phys. 58, 1200 (1980).

[48] Kai Nordlund (private communication).

[49] C. S. Becquart, M. F. Barthe, and A. De Backer, Phys. Scr. T 145, 014048 (2011).

[50] A. E. Sand, K. Nordlund, and S. L. Dudarev, J. Nucl. Mater. 455, 207 (2014).

[51] P. Pyykkö, Annu. Rev. Phys. Chem. 63, 45 (2012).

[52] P. Pyykkö, Chem. Rev. 112, 371 (2012). 